\title{
RESULTADOS POSTQUIRÚRGICOS EN LA CIRUGÍA COMBINADA DE GLAUCOMA. COMPARACIÓN DEL IMPLANTE EXPRESS CON LA TRABECULECTOMÍA ESTÁNDAR
}

\section{POSTOPERATIVE OUTCOMES AFTER COMBINED GLAUCOMA SURGERY. COMPARISON OF EX-PRESS MINIATURE IMPLANT WITH STANDARD TRABECULECTOMY}

\author{
GALLEGO-PINAZO R ${ }^{1}$, LÓPEZ-SÁNCHEZ E², MARÍN-MONTIEL J²
}

\section{RESUMEN}

Objetivos: Evaluar los hallazgos postquirúrgicos y el éxito tras cirugía combinada mediante facoemulsificación e implante Ex-PRESS comparado con cirugía combinada con trabeculectomía estándar.

Métodos: En esta serie prospectiva de 40 ojos consecutivos comparamos 20 ojos de 17 pacientes tratados mediante facoemulsificación combinada con cirugía filtrante de glaucoma con implante de válvula Ex-PRESS bajo un colgajo escleral con 20 ojos controles en 20 enfermos que fueron sometidos a facoemulsificación combinada con trabeculectomía estándar.

Resultados: El tiempo de seguimiento medio fue de 9,7 meses (rango: 4,5-15) en el grupo Ex-PRESS y 10,3 meses (rango 3,5-14,5) en el grupo trabeculectomía. La PIO media fue significativamente superior en el postoperatorio precoz en el grupo ExPRESS comparada con el de trabeculectomía. La tasa de complicaciones en dicho período resultó superior en el grupo sometido a trabeculectomía. No se objetivaron diferencias significativas en la tasa de éxito entre ambos grupos más allá de la primera semana.

\begin{abstract}
Purpose: To evaluate postoperative outcomes and success after combined phacoemulsification and glaucoma surgery with Ex-PRESS miniature implant compared with combined surgery with standard trabeculectomy.

Methods: In this prospective series of 40 consecutive eyes we compared 20 eyes in 17 patients treated with combined phacoemulsification and glaucoma filtering surgery with the Ex-PRESS miniature glaucoma implant under a scleral flap with 20 matched control eyes in 20 patients who underwent combined cataract and glaucoma surgery with trabeculectomy.

Results: The average follow-up was 9.7 months (range 4.5 to 15) for the Ex-PRESS group and 10.3 months (range 3.5 to 14.5) for the trabeculectomy group. The mean IOP was significantly higher in the early postoperative period in the Ex-PRESS group compared with the trabeculectomy group. Complications rate in the early postoperative period was significantily higher in the trabeculectomy group. No significant differences were objectified in success between both groups after the first week.
\end{abstract}

\footnotetext{
Recibido: 22/1/08. Aceptado: 8/6/09.

${ }^{1}$ Hospital Universitario La Fe de Valencia. Valencia. España.

2 Hospital Universitario Arnau de Vilanova de Valencia. Valencia. España.

Correspondencia:

Roberto Gallego Pinazo

Plaza Ciudad de Brujas, 4; 5

46001-Valencia

España

E-mail: robertogallego@comv.es
} 
Conclusiones: El implante Ex-PRESS constituye una alternativa eficaz y segura a la trabeculectomía en casos seleccionados que permite disminuir notablemente las clásicas complicaciones postquirúrgicas precoces asociadas a la trabeculectomía

Palabras clave: Cirugía combinada, cirugía filtrante de glaucoma, implante Ex-PRESS, trabeculectomía, complicaciones postquirúrgicas precoces.
Discussion: The Ex-PRESS implant is an effective and safe alternative to standard trabeculectomy in selected cases which makes possible to reduce remarkably the classic early postoperative complications associated with trabeculectomy (Arch Soc Esp Oftalmol 2008; 83: 293-298).

Key words: Combined surgery, glaucoma filtration surgery, Ex-PRESS miniature implant, trabeculectomy, early postoperative complications.

\section{INTRODUCCIÓN}

El implante Ex-PRESS (Optonol LTD, Kansas City, MO) es un tubo de acero inoxidable de $3 \mathrm{~mm}$ de longitud con una punta biselada de 27 Gauges de diámetro externo, una pestaña discoidal en el extremo proximal y una proyección en espolón que previene su extrusión. Este dispositivo no valvulado de drenaje fue diseñado expresamente para reducir la PIO en ojos con glaucoma. Asimismo su biocompatibilidad se evaluó en experimentos con conejos demostrando niveles de inflamación intraocular despreciables (1).

Los primeros estudios clínicos descubrieron la existencia de complicaciones en casos en que la válvula era colocada en el espacio subconjuntival (2) y en consecuencia se desarrollaron nuevas investigaciones a fin de disminuir estos problemas en base a la ubicación del Ex-PRESS por debajo de un colgajo escleral de espesor parcial $(3,4)$. En la actualidad la técnica quirúrgica idónea para implantar la válvula Ex-PRESS es muy similar a la de la trabeculectomía estándar, con la obvia excepción de que no es menester realizar ni una esclerotomía ni una iridectomía periférica (fig. 1).

En el presente estudio comparamos los hallazgos clínicos subsecuentes a la cirugía combinada de cristalino y glaucoma en pacientes con catarata y glaucoma con deficiente control tensional en los que se realiza un implante Ex-PRESS protegido por un colgajo escleral con aquéllos en que se realiza una trabeculectomía convencional.

\section{SUJETOS, MATERIAL Y MÉTODOS}

Llevamos a cabo un estudio prospectivo realizado en un único centro hospitalario y un único cirujano,

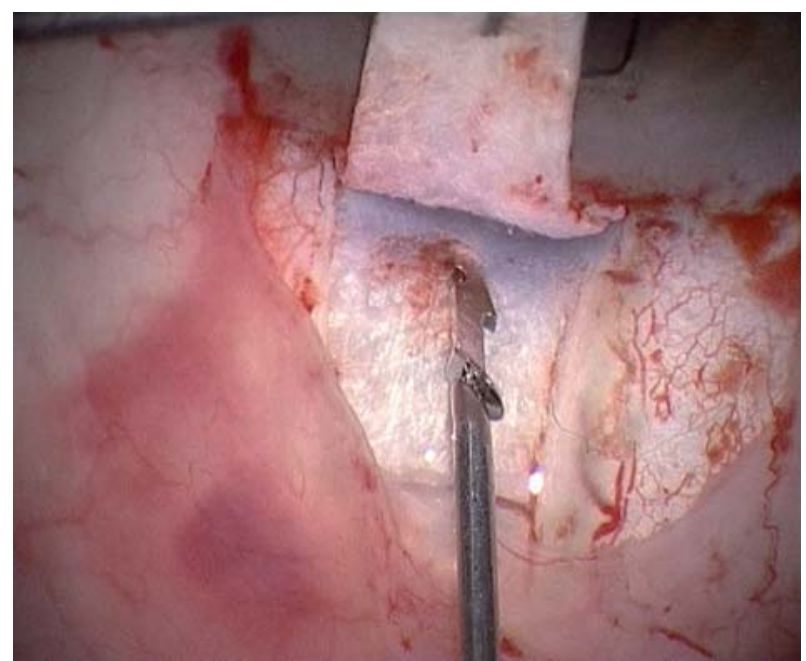

Fig. 1: Implante de un dispositivo Ex-PRESS bajo un colgajo escleral de espesor parcial rectangular de base en limbo.

seleccionando un total de 37 pacientes consecutivos que presentaran disminución de visión asociada a catarata y glaucoma con deficiente control tensional sin intervenciones quirúrgicas previas. Estos pacientes fueron asignados a dos grupos de estudio: cirugía combinada con implante de válvula Ex-PRESS y cirugía combinada con trabeculectomía.

De acuerdo con estudios previos (5) los criterios de selección para todos los integrantes de la muestra fueron: edad superior a 18 años; catarata susceptible de facoemulsificación; conjuntiva superior movilizable en al menos 3 horas de reloj; e indicación médica de cirugía de glaucoma en ausencia de tratamientos quirúrigicos previos (incluyendo iridotomías o trabeculoplastias).

Se registraron los datos preoperatorios referidos a sexo, edad, tipo y estado del glaucoma, opacidad de cristalino, PIO medida con tonómetro de aplana- 
ción y mejor agudeza visual corregida (MAVC). La información postquirúrgica incluyendo PIO, necesidad de colirios antiglaucomatosos, MAVC y complicaciones de la cirugía, fue recogida en los días postoperatorios $1,3,7$ y 15 , y posteriormente en los meses $1,2,6,12$ y 15 .

Como criterios de éxito quirúrgico consideramos: PIO mayores de $5 \mathrm{mmHg}$ y menores de $21 \mathrm{mmHg}$ sin colirios y sin posteriores reintervenciones (incluyendo la ciclofotocoagulación); y la ausencia de complicaciones que requiriesen el explante del ExPRESS (6). La necesidad de suturolisis o needling no fue considerada como fracaso de la cirugía.

Los pacientes del estudio fueron incluidos a lo largo de dos años y ambos grupos fueron equiparados en variables de edad y sexo. Todos los enfermos incorporados a la muestra tuvieron un tiempo mínimo de seguimiento postoperatorio de 3 meses.

En el grupo de 17 pacientes sometidos a facoemulsificación con implante de Ex-PRESS la edad media fue de 75,01 años con un total de 9 mujeres y 8 varones; en el grupo de 20 pacientes sometidos a facoemulsificación y trabeculectomía la edad media fue de 76,35 años con un total de 11 mujeres y 9 varones (tabla I). No encontramos diferencias

Tabla I. Parámetros descriptivos de ambos grupos de estudio: tipo de glaucoma subyacente, edad y sexo

\begin{tabular}{lccccc}
\hline \multicolumn{2}{c}{ Implante EX-PRESS } & \multicolumn{3}{c}{ Trabeculectomía } \\
\hline Glaucoma & Edad & Sexo & Glaucoma & Edad & Sexo \\
& & & & & \\
GPAA & 75 & $\mathrm{~V}$ & GPAA & 88 & $\mathrm{M}$ \\
GPEX & 81 & $\mathrm{M}$ & GPAA & 77 & $\mathrm{M}$ \\
GPAA & 72 & $\mathrm{~V}$ & GPAA & 76 & $\mathrm{~V}$ \\
GPAA & 76 & $\mathrm{M}$ & GPAA & 66 & $\mathrm{M}$ \\
GPEX & 85 & $\mathrm{M}$ & GPAA & 85 & $\mathrm{M}$ \\
GPEX & 75 & $\mathrm{~V}$ & GPEX & 75 & $\mathrm{~V}$ \\
GPAA & 84 & $\mathrm{M}$ & GPAA & 72 & $\mathrm{~V}$ \\
GPAA & 70 & $\mathrm{M}$ & GPAA & 87 & $\mathrm{M}$ \\
GPAA & 72 & $\mathrm{M}$ & GPAA & 76 & $\mathrm{~V}$ \\
GPAA & 88 & $\mathrm{M}$ & GPAA & 86 & $\mathrm{M}$ \\
GPAA & 72 & $\mathrm{M}$ & GPEX & 72 & $\mathrm{M}$ \\
GPAA & 54 & $\mathrm{M}$ & GPAA & 60 & $\mathrm{~V}$ \\
GPEX & 73 & $\mathrm{~V}$ & OTRO & 83 & $\mathrm{~V}$ \\
GPAA & 72 & $\mathrm{~V}$ & GPEX & 77 & $\mathrm{~V}$ \\
GPAA & 82 & $\mathrm{~V}$ & GPAA & 72 & $\mathrm{~V}$ \\
GPEX & 74 & $\mathrm{~V}$ & GPAA & 77 & $\mathrm{M}$ \\
GPAA & 71 & $\mathrm{~V}$ & GPAA & 69 & $\mathrm{M}$ \\
& & & GPEX & 81 & $\mathrm{M}$ \\
& & & GPAA & 73 & $\mathrm{~V}$ \\
& & & GPAA & 75 & $\mathrm{M}$ \\
\hline \hline
\end{tabular}

GPAA: Glaucoma Primario de Ángulo Abierto; GPEX: Glaucoma Pseudoexfoliativo; V: Varón; M: Mujer. estadísticamente significativas en estos parámetros epidemiológicos.

Por su parte, las dos muestras estaban constituidas por diversas formas de glaucoma: el Glaucoma Primario de Ángulo Abierto suponía el $70 \%$ de los ojos en el grupo del Ex-PRESS y el $75 \%$ de los pacientes en el grupo de la trabeculectomía; reconocimos un Glaucoma Pseudoexfoliativo en el 25\% del primer grupo y en el $20 \%$ del segundo.

Para el procesamiento estadístico de los datos del estudio fue empleado el programa informático SPSS 11.5 aplicando la prueba t-Student para muestras independientes.

\section{RESULTADOS}

No se evidenciaron diferencias significativas preoperatorias entre ambos grupos de estudio a la hora de evaluar la PIO ( $>0,79)$ ni el número de fármacos instilados como tratamiento hipotensor ( $p>0,67)$. La tabla II resume la estadística descriptiva de estos parámetros.

En la evolución postoperatoria de la PIO se reconocieron diferencias estadísticamente significativas en el período precoz en tanto que las tonometrías en los días tras la intervención 1 ( $\mathrm{p}<0,001), 3$ ( $\mathrm{p}<0,003)$ y $7(\mathrm{p}<0,006)$ reflejan valores mayores en el grupo del implante Ex-PRESS (fig. 2). A partir del día 15 tras la cirugía $(p>0,89)$ desaparecen dichas diferencias. Estos datos se correlaciones clínicamente con la ausencia de complicaciones en el subgrupo de ExPRESS, mientras que los pacientes que fueron sometidos a cirugía combinada con trabeculectomía sí experimentaron problemas como hipotalamias (6 de los 20 enfermos, $30 \%$ ) o hipemas ( 8 de los 20 enfermos, $40 \%$ ) de severidad variable, así como desprendimiento coroideo en 3 de los 20 enfermos (15\%). La inflamación postoperatoria fue más importante en la muestra sometida a trabeculectomía.

Tabla II. Evaluación preoperatoria de los valores de PIO y del número de fármacos hipotensores instilados en ambos grupos de estudio (media muestral y desviación estándar de la muestra)

\begin{tabular}{lcrcc}
\hline & Grupo & Media & DS & Valor P \\
\hline PIO & EX-PRESS & 28,25 & 7,15 & $>0,79$ \\
& Trabe & 27,31 & 4,42 & \\
Fármacos & EX-PRESS & 2,65 & 0,49 & $>0,67$ \\
& Trabe & 2,58 & 0,59 & \\
\hline \hline
\end{tabular}




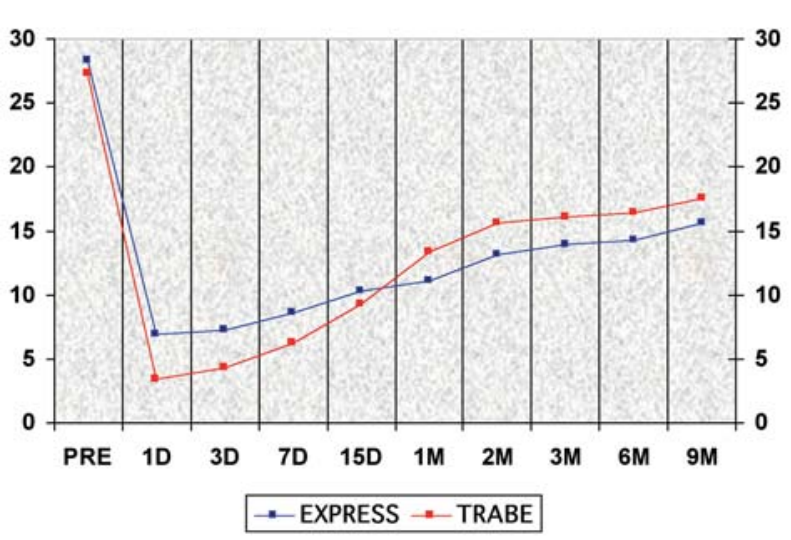

Fig. 2: Gráfico que representa la evolución de la PIO en ambos grupos desde el preoperatorio a lo largo de las revisiones postquirúrgicas. Existen diferencias significativas en el postoperatorio hasta el día 15, a partir del cual se pierde en adelante la significación estadística y el comportamiento es superponible en ambas muestras.

Por otra parte precisamos realizar suturolisis en 2 pacientes del grupo del Ex-PRESS y en 3 del grupo de la trabeculectomía, así como needling en 4 pacientes de la muestra del implante.

En lo que respecta al éxito quirúrgico de ambas muestras no se objetivaron diferencias significativas en el seguimiento a 15 meses entre los dos grupos de tratamiento $(p>0,21)$. Solamente en dos pacientes intervenidos mediante implante Ex-PRESS se necesitó introducir tratamiento con un fármaco para un eficiente control tensional, mientras que en sujetos sometidos a trabeculectomía fue necesario introducir tratamiento médico en tres pacientes con un fármaco y en otro más con dos fármacos para conseguir valores de PIO normales. Es decir, un 10\% de los ojos intervenidos con implante Ex-PRESS requirió tratamiento médico coadyuvante para controlar la PIO, mientras que este porcentaje es de un $20 \%$ en los ojos intervenidos mediante cirugía combinada con trabeculectomía.

\section{DISCUSIÓN}

En los últimos años el creciente interés en la cirugía no perforante del glaucoma ha impulsado este terreno dentro de la cirugía oftalmológica. La aparición de los implantes no valvulados Ex-PRESS suponen una alternativa importante a la hora de abordar la necesidad de intervenir a pacientes con glaucoma que no consiguen controlar las cifras ten- sionales con tratamiento médico y que no han sido sometidos a cirugías previas de glaucoma.

En nuestro trabajo, transcurrido un mes tras la cirugía encontramos PIO y tasas de éxito similares en ambos grupos de estudio. La tasa de éxito fue del $90 \%$ en la muestra Ex-PRESS frente al $80 \%$ en el grupo trabeculectomía, sin objetivar diferencias estadísticamente significativas. El número de colirios antiglaucomatosos tras la cirugía empleados tampoco mostró significación estadística.

En el período postoperatorio precoz, desde el día 1 hasta el día 7, la PIO fue significativamente menor en el grupo trabeculectomía frente a la muestra en que se realizara el implante Ex-PRESS bajo colgajo escleral. Las hipotonías postquirúrgicas mostraron avidez por el grupo trabeculectomía. Todos estos datos sugieren que el lumen de $50 \mu \mathrm{m}$ del dispositivo produce una resistencia adicional al flujo del acuoso durante los primeros días del postoperatorio. En ninguno de los ojos de nuestro estudio se objetivaron complicaciones del tipo erosión escleroconjuntival o exposición del implante, situaciones éstas frecuentes con la técnica de implantación subconjuntival (6-8).

A la luz de los datos ofrecidos en el presente estudio podemos sugerir que en pacientes sometidos a cirugía combinada el implante Ex-PRESS consigue un efecto a medio plazo superponible al conseguido por la trabeculectomía en el control de la PIO (9), pero con una reducción notable de las complicaciones precoces típicas de ésta como las hipotalamias, los hipemas o los desprendimientos coroideos.

\section{BIBLIOGRAFÍA}

1. Nyska A, Glovinsky Y, Belkin M, Epstein Y. Biocompatibility of the Ex-PRESS miniature glaucoma drainage implant. J Glaucoma 2003; 12: 275-280.

2. Wamsley S, Moster MR, Rai S, Alvim HS, Fontanarosa J. Results of the use of the Ex-PRESS miniature glaucoma implant in technically challenging, advanced glaucoma cases: a clinical pilot study. Am J Ophthalmol 2004; 138: 1049-1051.

3. Dahan E, Carmichael TR. Implantation of a miniature glaucoma device under a scleral flap. J Glaucoma 2005; 14: 98-102.

4. Coupin A, Li Q, Riss I. Ex-PRESS miniature glaucoma implant inserted under a scleral flap in open-angle glaucoma surgery: a retrospective study. J Fr Ophtalmol 2007; 30: $18-23$.

5. Maris PJ Jr, Ishida K, Netland PA. Comparison of trabeculectomy with Ex-PRESS miniature glaucoma device 
implanted under scleral flap. J Glaucoma 2007; 16: 1419.

6. Stewart RM, Diamond JG, Ashmore ED, Ayyala RS. Complications following Ex-PRESS glaucoma shunt implantation. Am J Ophthalmol 2005; 140: 340-341.

7. Rivier D, Roy S, Mermoud A. Ex-PRESS R-50 miniature glaucoma implant insertion under the conjunctiva combined with cataract extraction. J Cataract Refract Surg 2007; 33: 1946-1952.
8. Stein JD, Herndon LW, Brent Bond J, Challa P. Exposure of Ex-PRESS Miniature Glaucoma Devices: case series and technique for tube shunt renoval. J Glaucoma 2007; 16: 704-706.

9. Traverso CE, De Feo F, Messas-Kaplan A, Denis P, Levartovsky S, Sellem E, et al. Long term effect on IOP of a stainless steel glaucoma drainage implant (Ex-PRESS) in combined surgery with phacoemulsification. Br J Ophthalmol 2005; 89: 425-429. 
